\title{
Antibiotic Resistance Pattern in Healthy Children Diagnosed with Community Acquired Respiratory Tract Infections in King Abdulaziz University Hospital
}

Jawaher Walid ${ }^{1}$, Leena Moshref ${ }^{1}$, Mashael Alzanbagi ${ }^{1}$, Rana Moshref ${ }^{1}$, WejdanBaabdullah ${ }^{1}$, Eman Aldigs ${ }^{1,2}$, Turky AlAhmadi ${ }^{1,3}$, Jameela Kari ${ }^{1,3}$, Samah Alharbi $^{1}$, Osama Safdar ${ }^{1,3^{*}}$ and Osama Felemban ${ }^{1,3}$

${ }^{1}$ Faculty of Medicine, King Abdulaziz University, Jeddah, Saudi Arabia

${ }^{2}$ Department of Microbiology, Faculty of Medicine, King Abdulaziz University, Jeddah, Saudi Arabia

${ }^{3}$ Department of Pediatric, University Hospital, Faculty of Medicine, King Abdulaziz University, Jeddah, Saudi Arabia

*Corresponding author: Osama Safdar, Department of Pediatric Faculty of Medicine, King Abdulaziz University Hospital, King Abdulaziz University, Jeddah, Saudi Arabia, Tel: 96612 6408222 20208; Fax: 966126408353; E-mail: safderosama@hotmail.com

Rec Date: May 16, 2016; Acc Date: Jun 09, 2016; Pub Date: June 152016

Copyright: (c) 2016 Walid J, et al. This is an open-access article distributed under the terms of the Creative Commons Attribution License, which permits unrestricted use, distribution, and reproduction in any medium, provided the original author and source are credited.

\begin{abstract}
Objectives: To identify the prevalent organisms associated with respiratory tract infections according to age groups. To identify the most common antibiotics prescribed among pediatric age groups.

Methods: A retrospective cohort study conducted on patients aged 0-18 years, admitted to the inpatient department (IPD) diagnosed with respiratory tract infection between Jan 12009 to Jan 312015 in King Abdulaziz University Hospital (KAUH). Demographic information, diagnosis, date of admission, laboratory investigations, and treatment were collected from patients' electronic and paper records then analyzed. Exclusion criteria were based on congenital defects, chronic diseases, and compromised immunity.
\end{abstract}

Results: 122 patients with respiratory tract infections were selected for the study (73 males and 49 females). $45.9 \%$ of which were infants-toddlers $(n=56)$. The most common diagnosis was pneumonia unspecific $(n=32)$, followed by acute tonsillitis $(n=24)$ and otitis media $(n=22) .52$ microorganisms were isolated from clinical samples, $14 \%$ being Pseudmonas aeruginosa and 13\% Streptococcus pneumoniae. 171 antimicrobials were prescribed during the study period. Penicillins were the most frequent $(n=78)$, followed by Cephalosporins ( $n=45)$ and Macrolides $(n=22)$. $6.897 \%$ resistance was detected among 87 files with culture and sensitivity tests, $30 \%$ of which were positively cultured, ranking resistance with $20 \%$.

Conclusions: Despite the results showing minimal resistance, antibiotic resistant organisms remain a global concern that needs to be addressed to prevent the spread of antimicrobial resistant infections.

Keywords: Antibiotic; Resistance; Respiratory tract infection (RTI); Community acquired (CA)

\section{Introduction}

Antibiotic resistance has cast a shadow over the medical glory discovery, which we call "antibiotics". Ever since its discovery, antibiotics have been used as a treatment for patients with bacterial infections. However, these infectious organisms tend to show resistance to antibiotics due to overexposure and adaptation [1]. Resistant pathogens of the respiratory tract, such as $S$. pneumoniae, $H$. influenzae and M. catarrhalis, have been reported in Saudi Arabia [2]. The underlying complications of such problem make it a global threat [3]. The rate of treatment and recovery for antibacterial resistant infections will be prolonged, as well as increase the morality rate for patients with severe infections. In the United States, two million people annually acquire serious bacterial infections that are resistant to one or more antibiotics and at least 23,000 people die due to antibioticresistant infections [4]. In 2009, the European Medicines Agency (EMA) along with the European Centre for Disease Prevention and Control (ECDC) published that an estimation of 25,000 people die each year in Europe from antibiotic-resistant bacteria [5]. While in the USA, a study in 2005 estimated that 94,000 invasive MRSA (Methicillin-Resistant Staphylococcus aureus) infections required hospitalization and were associated with 19,000 deaths [6]. Another study conducted in Kano, Nigeria 2013, showed that the resistance of $S$. aureus to be only moderately sensitive to chlorampenicol, ceftazidine, ciproflaxacin and rocephine and at the same time showing resistance to augmentin, amoxicillin, erythromycin, tetracycline, gentamycin, cotrimoxazole and pefloxacin [7]. A major pathogen that has been causing a significant infection in children in Saudi Arabia is the $S$. aureus (CA-MRSA) bacteria [8]. The MRSA was also reported to be resistant to penicillin and cephalloxcinin in Assir Hospital-Abha, KSA [9]. With the increase in number of antibacterial resistant microorganisms, the efficacy of such drugs have been reduced or, in some cases, completely ineffective [10]. Although resistant infections are more common in individuals on long courses of antibiotic treatment; it is difficult to ascertain whether resistance is the cause of death or a correlate of long antibiotic treatment, hospitalization, and underlying sickness [1]. On the economic side, health care costs will be amplified due to expensive therapies and prolonged length-of-stay [11]. Due to the continuous pandemic emergence of resistance, we aim 
Citation: Walid J, Moshref L, Alzanbagi M, Moshref R, Baabdullah W, et al. (2016) Antibiotic Resistance Pattern in Healthy Children Diagnosed with Community Acquired Respiratory Tract Infections in King Abdulaziz University Hospital. J Med Microb Diagn 5: 232. doi: 10.4172/2161-0703.1000232

Page 2 of 7

to detect the antibiotic resistance pattern in healthy children diagnosed with community acquired respiratory tract infections in King Abdulaziz University Hospital, as well as the prevalent resistant organisms.

\section{Materials and Methods}

The study was carried out in King Abdulaziz University Hospital (KAUH), a teaching hospital situated in Jeddah, Saudi Arabia.

\section{Study design}

This was a retrospective cohort study, which included accessing patients' medical records from the Phoenix electronic filing system and paper files within a 5-year interval (Jan 2009 to Jan 2015) for patient profile (age, sex, date of admission), diagnosis, laboratory investigations, detectable organisms, antibiotic prescriptions based on clinical practice reference, and duration of therapy. The information was collected and analyzed in consultation with a Microbiologist, and a Pediatric Pulmonologist consultant.

\section{Hajj study design}

Hajj season was calculated based on the first day pilgrims arrive to Saudi Arabia and the last day they leave. Saudi Arabia begins to welcome pilgrims on the 1st of Thul-qidah [12]. The last day for the pilgrim to leave is on the 15th of Muharram [13]. Hajj seasons vary each year in the Gregorian calendar because of the 11-day difference in Hijri-Lunar calendars that Hajj follows (Table 1). This could also contribute as a factor in Hajj infection rate (Figure 1).

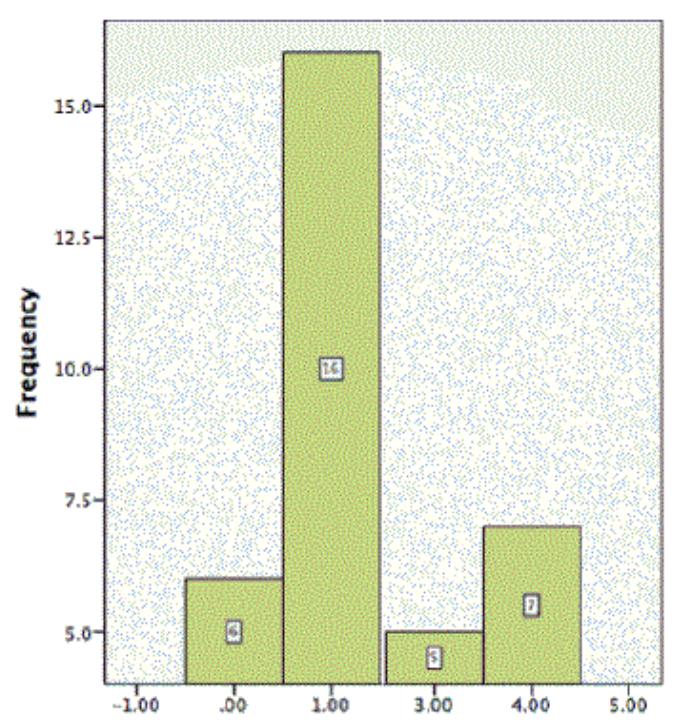

Figure 1: Frequency of RTIs in Hajj Seasons in KAUH.

27.9\% of all RTI were detected in Hajj season. Calculated Chisquare showed a positive correlation between hajj season and RTI rates with a $\mathrm{p}$ value of 0.023 .

\section{Duration of study}

The duration of study was 12 months (July 2014 to Sep 2015). The Biomedical Ethics Research Committee in King Abdulaziz University approved the study.

\begin{tabular}{|l|l|l|}
\hline Haj Season & Hijri Calendar & Gregorian Calendar* \\
\hline 2010 & $1 / 11 / 1431-15 / 1 / 1432$ & $09 / 10 / 2010-21 / 12 / 2010$ \\
\hline 2011 & $1 / 11 / 1432-15 / 1 / 1433$ & $29 / 09 / 2011-11 / 12 / 2011$ \\
\hline 2012 & $1 / 11 / 1433-15 / 1 / 1434$ & $17 / 09 / 2012-29 / 11 / 2012$ \\
\hline 2013 & $1 / 11 / 1434-15 / 1 / 1435$ & $07 / 09 / 2013-11 / 11 / 2013$ \\
\hline 2014 & $1 / 11 / 1435-15 / 1 / 1436$ & $27 / 08 / 2014-08 / 11 / 2014$ \\
\hline *Gregorian dates were calculated by using Makkah Calendar <http:// \\
www.makkahcalendar.org/ar/hijriCalendar.php> \\
\hline
\end{tabular}

Table 1: Hajj season dates.

\section{Study population}

The study was conducted on 122 out of 464 patients who visited the IPD during the 5-year period. The subjects were included on the basis of inclusion and exclusion criteria. All patients between the ages of 0-18 years old, hospitalized in $\mathrm{KAUH}$, with respiratory tract infections were studied. However, patients with congenital defects, chronic diseases, and compromised immunity were excluded.

\section{Outcome measures}

The outcome measures included types of respiratory tract infections in relation to age and gender, common organisms in different age groups, types and frequency of antibiotics prescribed, resistant organisms and their resistant drugs.

\section{Clinical samples analysis and sensitivity studies}

Obtained samples (blood, sputum, nasopharyngeal swab, oropharyngeal swab, urine, and skin) were ordered by the treating physician and analyzed by the VITEK 2 system (bioMérieux) 29 in the microbiology laboratory of the hospital, for bacterial identification and antibiotic susceptibility testing (AST).

\section{Statistical analysis}

Data were analyzed using the SPSS Software for Mac OSX version 21. Comparisons were done using Chi square with $\mathrm{P}<0.05$ showing significance.

\section{Results}

Out of 464 reviewed patient files, 122 met the criteria for the study, 73 (59.8\%) were male and $49(40.2 \%)$ were female. The highest numbers of patients were infants and toddler (0-2 years old) $(45.9 \%)$ and the lowest numbers of patients were teenagers (13-18 years old) (9.8\%). During the study, it was observed that the major age group exposed to respiratory tract infections was infants-toddler (56 cases), followed by preschoolers (39 cases), school age (15 cases) then adolescence (12 cases). The most commonly diagnosed respiratory tract infection was pneumonia unspecific (32 cases), of which 22 cases were infants-toddlers. Followed by, 24 cases of acute tonsillitis, half of 
Citation: Walid J, Moshref L, Alzanbagi M, Moshref R, Baabdullah W, et al. (2016) Antibiotic Resistance Pattern in Healthy Children Diagnosed with Community Acquired Respiratory Tract Infections in King Abdulaziz University Hospital. J Med Microb Diagn 5: 232. doi: 10.4172/2161-0703.1000232

which were in the preschooler age group (12 cases), and then 22 cases of Otitis Media (Table 2).

\begin{tabular}{|c|c|c|c|c|c|}
\hline \multirow[b]{2}{*}{ Diagnosis } & \multicolumn{4}{|l|}{ Age Group } & \multirow[b]{2}{*}{ Total } \\
\hline & Infants-Toddler & Preschooler & School age & Early adolescence & \\
\hline Pneumonia Unspecific & 22 & 5 & 3 & 2 & 32 \\
\hline Acute tonsillitis & 6 & 12 & 5 & 1 & 24 \\
\hline Otitis Media & 4 & 14 & 2 & 2 & 22 \\
\hline Viral pneumonia & 12 & 5 & 0 & 0 & 17 \\
\hline Bacterial Pneumonia & 8 & 1 & 3 & 0 & 12 \\
\hline Acute sinusitis unspecific & 1 & 1 & 2 & 6 & 10 \\
\hline Others & 3 & 1 & 0 & 1 & 5 \\
\hline Total & 56 & 39 & 15 & 12 & 122 \\
\hline
\end{tabular}

Table 2: RTIs distributed among different age groups.

Collected clinical samples revealed 52 isolated microorganisms. The most common bacterial pathogens were Pseudomonas aeruginosa (11.54\%), followed by Staphylococcus aureus (7.69\%), Streptococcus pneumoniae (7.69\%), Coagulase negative staphylococcus $(7.69 \%)$, and mixed culture (7.69\%). Other organisms accounted for (65.4\%). Most organisms were found in the infants-toddler age group by $(80.76 \%)$ (Table 3).
Patients' medical records showed that 171 antibiotics were prescribed over the period of 5 years; penicillins $(n=78)$ were the most frequently prescribed antibiotics, followed by cephalosporins $(n=48)$, macrolides $(n=22)$, aminoglycosides $(n=5)$ (Table 4$)$. The most commonly used drug of these classes was augmentin $n=56$ (penicillins), followed by Cephalosporins (ceftriaxone $\mathrm{n}=24$ and cefuroxime $\mathrm{n}=21$ ), and Macrolides (erythromycin $\mathrm{n}=15$ ) (Table 3).

\begin{tabular}{|c|c|c|c|c|c|}
\hline \multirow[t]{2}{*}{ Organism } & \multicolumn{4}{|l|}{ Age Groups } & \multirow{3}{*}{$\begin{array}{l}\text { Tota } \\
6\end{array}$} \\
\hline & Infants-Toddler & Preschooler & School age & Early adolescence & \\
\hline$P$. aeruginosa & 6 & 0 & 0 & 0 & \\
\hline Staphylococcus aureus & 2 & 0 & 0 & 2 & 4 \\
\hline Coagulase-negative staphylococci & 4 & 0 & 0 & 0 & 4 \\
\hline Mixed culture & 1 & 2 & 1 & 0 & 4 \\
\hline S.pneumoniae & 4 & 0 & 0 & 0 & 4 \\
\hline S. epidermidis & 3 & 0 & 0 & 0 & 3 \\
\hline Haemophilus Influenzae & 3 & 0 & 0 & 0 & 3 \\
\hline Klebsiella pneumoniae & 3 & 0 & 0 & 0 & 3 \\
\hline Diphtheroid species & 2 & 0 & 1 & 0 & 3 \\
\hline Streptococcus pyogenes & 0 & 2 & 0 & 0 & 2 \\
\hline Enterococcus faecium & 2 & 0 & 0 & 0 & 2 \\
\hline Staphylococcus hominis & 2 & 0 & 0 & 0 & 2 \\
\hline Enterobacter cloacae & 2 & 0 & 0 & 0 & 2 \\
\hline Candida albicans & 1 & 0 & 1 & 0 & 2 \\
\hline Aspergillus & 1 & 0 & 1 & 0 & 2 \\
\hline
\end{tabular}


Citation: Walid J, Moshref L, Alzanbagi M, Moshref R, Baabdullah W, et al. (2016) Antibiotic Resistance Pattern in Healthy Children Diagnosed with Community Acquired Respiratory Tract Infections in King Abdulaziz University Hospital. J Med Microb Diagn 5: 232. doi: 10.4172/2161-0703.1000232

Page 4 of 7

\begin{tabular}{|l|l|l|l|l|l|}
\hline Kocuria rosea & 1 & 0 & 0 & 0 & 1 \\
\hline Bordetella pertussis & 1 & 0 & 0 & 0 & 1 \\
\hline Respiratory syncytial virus (RSV) & 1 & 0 & 0 & 0 & 1 \\
\hline Moraxella catarrhalis & 1 & 0 & 0 & 0 & 1 \\
\hline Escherichia coli & 1 & 0 & 0 & 0 & 1 \\
\hline Unknown (miscellaneous) & 1 & 0 & 4 & 2 & 52 \\
\hline Total & 42 & 4 & & 1 \\
\hline
\end{tabular}

Table 3: Organism distribution of community acquired infection in healthy children.

Furthermore, findings have shown that a total of 98 patients (out of treatment, and $2.5 \%$ 4-drug treatment. Average antimicrobial 122) received antibacterial therapy. Of which, $40.98 \%$ received a single treatment was 1.8 -drug/case. antimicrobial treatment, $20.49 \%$ 2-drug treatment, $16.39 \%$ 3-drug

\begin{tabular}{|c|c|c|c|c|c|}
\hline Drug & Drug Prescribed & $\begin{array}{l}\text { Amount of } \\
\text { Prescription }\end{array}$ & $\%$ of Prescription & Amount of Resistance & Resistance \% \\
\hline \multirow[t]{9}{*}{ Penicillins } & Augmentine & 56 & $32.7 \%$ & Undetermined & \\
\hline & Ampicillin & 10 & $5.8 \%$ & 2 & $1.16 \%$ \\
\hline & Tazobactem & 4 & $2.3 \%$ & & \\
\hline & Piperacillin & 4 & $2.3 \%$ & 1 & $0.575 \%$ \\
\hline & Cloxacillin & 2 & $1.2 \%$ & & \\
\hline & Penicillin G & 1 & $0.6 \%$ & 1 & $0.6 \%$ \\
\hline & Amoxacillin & 1 & $0.6 \%$ & & \\
\hline & Oxacillin & 0 & & 2 & Infinity \\
\hline & Total & 78 & $45.6 \%$ & & \\
\hline \multirow[t]{4}{*}{ Cephalosporins } & Ceftriaxone & 24 & $14.0 \%$ & Ceftriaxone & 24 \\
\hline & Cefuroxime & 21 & $12.3 \%$ & Cefuroxime & 21 \\
\hline & Cefotaxime & 3 & $1.8 \%$ & Cefotaxime & 3 \\
\hline & Total & 48 & $28.1 \%$ & Total & 48 \\
\hline \multirow[t]{4}{*}{ Macrolides } & Erythromycin & 15 & $8.8 \%$ & 3 & $1.76 \%$ \\
\hline & Clarithromycin & 6 & $3.5 \%$ & & \\
\hline & Azithromycin & 1 & $0.6 \%$ & & \\
\hline & Total & 22 & $12.9 \%$ & & \\
\hline \multirow[t]{4}{*}{ Aminoglycosides } & Gentamycin & 3 & $1.7 \%$ & & \\
\hline & Amikacin & 1 & $0.6 \%$ & & \\
\hline & Streptomycin & 1 & $0.6 \%$ & & \\
\hline & Total & 5 & $2.9 \%$ & & \\
\hline \multirow[t]{2}{*}{ Antiviral } & Tamiflu (Oseltamevir) & 4 & $2.3 \%$ & & \\
\hline & Acyclovir & 1 & $0.6 \%$ & & \\
\hline
\end{tabular}


Citation: Walid J, Moshref L, Alzanbagi M, Moshref R, Baabdullah W, et al. (2016) Antibiotic Resistance Pattern in Healthy Children Diagnosed with Community Acquired Respiratory Tract Infections in King Abdulaziz University Hospital. J Med Microb Diagn 5: 232. doi: 10.4172/2161-0703.1000232

Page 5 of 7

\begin{tabular}{|l|l|l|l|l|l|}
\hline & Total & 5 & $2.9 \%$ & & \\
\hline Nitroimidazoles & Metronidazole & 4 & $2.3 \%$ & & \\
\hline Glycopeptide & Vancomycine & 3 & $1.8 \%$ & Undetermined & \\
\hline Lincosamides & Clindamycine & 3 & $1.8 \%$ & 1 & $0.6 \%$ \\
\hline Fluoroquinolones & Ciprofloxacin & 2 & $1.2 \%$ & & \\
\hline Antifungal & Nystatin & 1 & $0.6 \%$ & & \\
\hline
\end{tabular}

Table 4: Frequency of Antimicrobials Prescribed and their resistance percentages $(n=171)$.

Antibiotics were most commonly used in unspecified pneumonia cases (33.9\%), followed by viral pneumonia (17.5\%) and acute tonsillitis (14.6\%). Augmentin was the most commonly prescribed antibiotic for pneumonia cases (regardless of its type), followed by ceftriaxone and ampicillin. Almost $44 \%$ of antibiotic prescribed drugs were for pneumonia patients ( $31 \%$ unspecified, $11 \%$ bacterial, and $2 \%$ bronchopneumonia). Multiple drug therapy was also noticed in $60 \%$ of these cases the most commonly prescribed antibiotic in infancy, preschool, and school age is Augmentin (Figure 2).

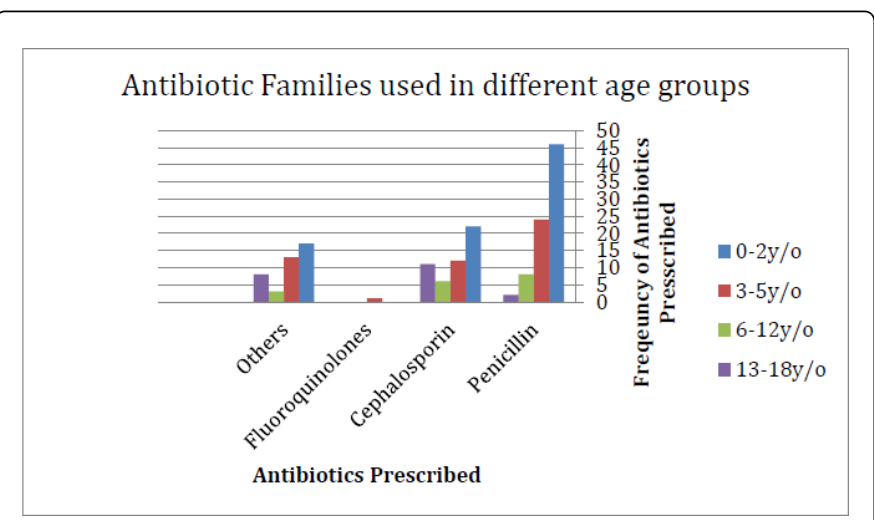

Figure 2: Antibiotic Families used in different age groups.

This chart illustrates the frequency of antimicrobial classes prescribed in different age groups. Others include: Macrolides, Nitromidazole, Aminoglycosides, Vancomycin, Antiviral, Antifungal and Rifampicin.

\section{Resistance of organisms according to culture and sensitivity test}

Tests have shown a resistance of $6.897 \%$ among 87 files with culture and sensitivity ordered, those of which 30 were positively cultured, ranking resistance with $20 \%$. Klebsiella pneuminiae, Pseudomonas aeruginosa, Enterococcus faecium, Staphylococcus aureus and $S$. epidermidis showed antimicrobial resistance to penicillin family (ampicillin, oxacillin, pipracillin, benzylpeniciilin), macrolides family (clindamycin), aminoglycoside family (erythromycin) and nitrofurantoin. Staphylococcus epidermidis, Enterococcus Faecium, Klebsiella pneumoniae and Pseudomonas showed resistance to the penicillin family $(\mathrm{n}=6)$. While Pseudomonas and Staphylococcus aureus showed resistance to erythromycin $(\mathrm{n}=3)$, Enterococcus faecium showed resistance to nitrofuratoin $(\mathrm{n}=1)$ and Staphylococcus epidermidis showed resistance to clindamycin $(\mathrm{n}=1)$.
Furthermore, males have shown great resistance compared to females by a ratio of 3.28:1.34. Resistance was highest in erythromycin scoring $1.76 \%$ among $15 \%$ of total prescriptions, followed by ampicillin scoring $1.16 \%$ among $5.8 \%$ of total prescriptions. While, clindamycin and penicillin scored $0.6 \%$ each, from $1.8 \%$ and $0.6 \%$ of total prescriptions respectively, followed by piperacillin, which showed a resistance of $0.575 \%$ from $2.3 \%$ of total prescriptions.

\section{Discussion}

Previous studies had implicated Haemophilus influenzae, Staphylococcus aureus, Streptococcus pneumoniae, Klebsiella pneumoniae, and Streptococcus pyogenes as the main common bacterial pathogens for RTIs [14]. Our findings were in harmony with these studies, in terms of Staphylococcus aureus (7.69\%) and Streptococcus pneuminae (7.69\%). In contrast, Haemophilus influenzae, Klebsiella pneumoniae, Streptococcus pyogenes were not as common in our results.

According to the WHO, Pseudomonas aeruginosa may also be implicated [15], which was the primary finding in this research. In other developing countries; the most common pathogen is $S$. pneumoniae [16]. Which has been reported to vary from $9 \%-72 \%$ in different studies in sub-Saharan Africa [17].

Furthermore, colonization by $S$. pneumoniae, $H$. influenzae, $M$. catarrhalis, and $S$. aureus are common in respiratory tract infections under healthy circumstances, but higher colonization rates were noticed in children [18]. This would support our findings that showed that younger age groups, specifically infants and toddlers (0-2 years old), were at a higher risk than older age groups. A community-based study in Gambia, Hill et al. also documented a higher prevalence of respiratory pathogens in young children than in adults [17], as well as a study conducted in Nepal showed that 1-10 years old were at a higher risk than other age groups [19]. Respiratory infections were responsible for $74.4 \%$ of antibiotic prescriptions [20]. Antibiotic frequency was evident in the study with penicillin (augmentin) being the most frequently prescribed antibiotic, followed by cephalosporins (ceftriaxone, and cefuroxime). Penicillin is the highest prescribed cause of it is increase activity against most Gram-positive cocci, Grampositive rods, Gram-negative cocci, and anaerobes. Exceptions are bacteria that acquired resistance to penicillin as well as certain anaerobes such as bacteroides that produce beta-lactamase. Also, research conducted in 19 European countries "Variation in pediatric hospital antibiotic guidelines in Europe" agreed that penicillin and amoxicillin were the antibiotics most commonly recommended for respiratory tract infections (RTIs) (up to 76\%), cephalosporin for urinary tract infection (UTI) (up to 50\%) [21]. 
Citation: Walid J, Moshref L, Alzanbagi M, Moshref R, Baabdullah W, et al. (2016) Antibiotic Resistance Pattern in Healthy Children Diagnosed with Community Acquired Respiratory Tract Infections in King Abdulaziz University Hospital. J Med Microb Diagn 5: 232. doi: $10.4172 / 2161-0703.1000232$

Page 6 of 7

Furthermore, this research results have shown that infants/toddlers $(0-2 y / 0)$ had the highest number of prescribed antibiotics of about 85 prescriptions out of 171 of total prescriptions of all age groups. Penicillin prescriptions were 46 prescriptions/85 prescriptions, followed by cephalosporin of about 22 prescriptions/ 85 prescriptions in this age group.

With more than 122 observed cases were affected by upper respiratory tract (frontal sinuses, tonsillitis, pharyngitis,) or lower respiratory tract Infection (bronchitis, pneumonia, influenza). $46 \%$ of these cases were infant and toddler; this could be related to high prevalence rate of community-acquired pneumonia. Respiratory syncytial virus, the most common cause of bronchiolitis and lower respiratory tract infections in the first year of life in comparison to other studies [22], was found to be $2 \%$ among infants and toddler.

Vaccination has markedly decreased the rate of respiratory infections such as pertussis (only found in one case), measles, $H$. influenzae, and pneumococcus in all cases.

Almost all cases of staphylococcal infection targeted the lower respiratory tract, which caused pneumonia and bronchopneumonia in $3 \%$ of infants and toddlers.

Most of the acute tonsillitis $79.1 \%$ weren't cultured, $8 \%$ of the acute tonsillitis cases were positive for Streptococcus pyogenes. The result agrees with other study that linked $S$. pyogenes with recurrent infection of tonsillitis [23]. The remaining results were $8 \%$ of other organisms and $4.9 \%$ for mixed culture. Negatively cultured results were excluded from the study, and no further viral tests were performed.

Antibiotic resistant microorganisms were found in this study with a (6.897\%) resistance in accordance to culture/sensitivity test. Staphylococcus aureus being the most resistant followed by $K$. pneumoniae, $P$. aeruginosa, E. faecium, and $S$. epidermidis. While a study in 2013 showed that the resistant pathogens of the respiratory tract in Saudi Arabia were $S$. pneumoniae, $H$. influenzae and $M$. catarrhalis [2]. Furthermore, the antibiotic resistant organisms were predominant in the infant age group (mainly 1 year olds), except for 1 case that was 14 years old showed resistance. This could be explained by a study conducted by Ilan Dalal, that showed fetal serum immunoglobulin differed in stages of infancy. In utero, fetal serum immunoglobulin concentrations are low and at birth levels are equal or slightly higher than maternal serum IgG levels. At three months of age, IgG level gradually falls followed by progressive increase and is called physiologic hypogammaglobulinemia of infancy [24].

$S$. epidermidis, E. faecium, $K$. pneumoniae, and Pseudomonas showed a resistance of $54.55 \%$ to penicillin family. Pseudomonas and Staphylococcus aureus showed a resistance of $27.7 \%$ to erythromycin. $E$. faecium and $S$. epidermidis showed a resistance of $9.09 \%$ to nitrofuratoin and clindamycin respectively. While a study in Warsaw, Poland, showed that there was a $100 \%$ resistance of $K$. pneumoniae to netilmicin and amikacin in neonates, and detected methicillin resistant $S$. aureus strains in $2.7 \%$ of cases [25]. According to a study conducted by Mayanskiy N, Alyabieva N in Morocco and Russia showed that the rate of multidrug-resistant pneumococci to more than 3 antibiotics was $22 \%$. Penicillin non-susceptibility was displayed by $28 \%$, while the resistance rate to erythromycin was 26\%. [26]. Another study supported the previous statement, the antibiotic resistance rates in China of the $S$. pnuemonia isolates to erythromycin, clindamycin and tetracycline were $95.9 \%, 94.5 \%, 87.7 \%$ [27].
Despite having the study carefully carried out, some limitations were present. The research was conducted in a tertiary healthcare governmental center with an inpatient sample population only, thus limiting the sample size and follow up. A sample population from outpatient clinics would show greater flow of patients and simple antibiotic prescription. Moreover, the sample size was considerably small in comparison to the time duration chosen (5 years), this was due to the exclusion criteria, which excluded many patients with congenital heart defects, chronic diseases, and known/unknown syndromes. Another reason was missing patient files and incomplete files caused by two floods that affected Jeddah in 2009 and 2011; limiting files to only 2 in 2009 and 39 in 2011. Additionally, the electronic database was first launched in 2012, but has officially been in use since 2014; which lead to accessing both electronic and paper files for data collection, leading to extending the length to conduct the study and should note that the diagnosis coding system was markedly specific making it difficult to extract all case files. Lastly, patients' electronic files are not enlisted in the national registry in Saudi Arabia, making it more difficult to track patients' health status throughout the 5 years.

\section{Hajj studies}

Respiratory tract infection during Hajj (pilgrimage to Mecca) is a common illness, KKKK AUH is not directly responsible for treating pilgrims, but the trend of infection is positively higher during Hajj season. This could be due to the close contacts among the pilgrims, as well as the crowding; the potential for transmission of infections is expected to be high.

These pilgrims can be a source for spreading infection on their return home. Although vaccination program is implemented, its efficacy is uncertain in this religious season [28]. Future studies should concentrate on prevention and mitigation of these infections. The results agree with other studies conducted during hajj season.

\section{Conclusion}

Despite the limited number of isolates used in the study, minimal antibacterial resistance was present. Nonetheless, antibacterial resistance remains a global concern that needs to be addressed to prevent the spread of antimicrobial resistant infections. Infants and toddlers are at a higher risk than older age groups. Penicillins followed by cephalosporins remain to be the drug of choice for the treatment of many respiratory tract infections.

\section{Recommendations}

The usage of rapid diagnostic tests should be implemented since they have the potential to detect a wider range of cases with respiratory tract infections with greater sensitivity (Pavia AT, 2011), as well as the usage of International guidelines on antibiotic prescription rather than clinical proficiency. Furthermore, Future researches are recommended to investigate common organisms in Umrah periods, and additionally find a relationship between infants and high resistance patterns. The need for antibiotic resistance monitoring is vital in this era. In addition, we are hoping for an electronic filing system to completely replace the traditional paper system that can be part of the national registry among healthcare facilities. 
Citation: Walid J, Moshref L, Alzanbagi M, Moshref R, Baabdullah W, et al. (2016) Antibiotic Resistance Pattern in Healthy Children Diagnosed with Community Acquired Respiratory Tract Infections in King Abdulaziz University Hospital. J Med Microb Diagn 5: 232. doi: 10.4172/2161-0703.1000232

Page 7 of 7

\section{Acknowledgements}

All the authors declare that they have no conflict of interest with the data presented in this manuscript. Authors would like to extend their gratitude to Dr. Bakr Al Siddiq, for his guidance in statistical analysis and usage.

\section{References}

1. Centers for Disease Control and Prevention. "About Antimicrobial Resistance: A Brief Overview." Accessed on 14 June 2016.

2. Shariff A, Al Dhubiab B, Al Nowis M, Al Haddad F, Al Omran N, et al. (2013) "Statistical Investigation Of Patient Factor In Development Of Antibiotc Resistance - A Study In Eastern Province Of Saudi Arabia." Int J Pharm Pharm Sci 5: 2013.

3. World Health Organization "WHO's first global report on antibiotic resistance reveals serious, worldwide threat to public health". Accessed on 14 June 2016.

4. Centers for Disease Control and Prevention. "Antibiotic resistance threats in the United States, 2013". Accessed on 14 June 2016.

5. ECDC/EMEA joint technical report "The bacterial challenge: time to react". Accessed on 14 June 2016.

6. Norrby R, Powell M, Aronsson B, Monnet D, Lutsar I, et al. (2009) European Centre for Disease Prevention and Control. Tech. no. 10.2900/2518. European Medicines Agency. Accessed on 14 June 2016.

7. Taura D, Hassan A, Yayo A, Takalmawa H (2013) "Bacterial Isolates of the Respiratory Tract Infection and Their Current Sensitivity Pattern among Patients Attending Aminu Kano Teaching Hospital Kano-Nigeria." IJRM 4: 226-31.

8. Bukhari EE, Al-Otaibi FE (2009) "Severe community-acquired infection caused by methicillin-resistant Staphylococcus aureus in Saudi Arabian children." Saudi Med J 30: 1595-10600.

9. Hamid ME (2011) "Resistance pattern of coagulase positive Staphylococcus aureus clinical isolates from Asir region, Kingdom of Saudi Arabia”. JMA 3: 102-108.

10. Frieden $\mathrm{T}$ (2013) "Antibiotic Resistance Threats in the United States, 2013" CDC Annual report 4-5.

11. Emanuele P (2010) "Antibiotic resistance." National Center for Biotechnology Information. U.S. National Library of Medicine.

12. (2014) "Hajj Organization." Commission of Hajj and Umrah. Accessed on 14 June 2016.

13. (2015)"Hajj Visa." Ministry of Foreign Affairs. Accessed on 14 June 2016.

14. Lykova EA, Vorob'ev AA, Bokovoi AG, Karazhas NV, Evseeva LF (2003) Associated infections in acute bronchopulmonary infections in children. Vestn Ross Akad Med Nauk 6: 9-12.
15. Ndip RN, Aroke G, Mbacham W, Ndip LM, Titanji VP (2003) Antibiogram and plasmid profiles of Neisseria gonorrhoeae isolates from Cameroon: useful tools for epidemiological survey. Afr J Reprod Health 7: 100-105.

16. Herruzo R, Chamorrol L, García ME, González MC, López AM, et al. (2002) Prevalence and antimicrobial-reistance of S. pneumoniae and S. pyogenes in healthy children in the region of Madrid. Int J Pediatr Otorhinolaryngol 65: 117-123.

17. Hill PC, Akisanya A, Sankareh K, Cheung YB, Saaka M, et al. (2006) Nasopharygeal carriage of Streptococcus pneumoniae in Gambian villagers. Clin Infect Dis 43:673-679.

18. Bosch A, Biesbroek G, Trzcinski K, Sanders E, Bogaert D (2013) Viral and Bacterial Interactions in the Upper Respiratory Tract. PLoS Pathog 9: p.e1003057.

19. Khan S, Priti S, Ankit S (2015) Bacteria Etiological Agents Causing Lower Respiratory Tract Infections and Their Resistance Patterns. Iran Biomed J 19:240-246.

20. O' Brien K, Bellis TW, Kelson M, Hood K, Butler CC, et al. (2015) "Clinical predictors of antibiotic prescribing for acutely ill children in primary care: an observational study." Br J Gen Pract 65:e585-592.

21. Spyridis N, Syridou G, Goossens H, Versporten A, Kopsidas J, et al. (2015) ARPEC Project Group Members. "Variation in paediatric hospital antibiotic guidelines in Europe." Arch Dis Child. archdischild-2015-308255.

22. Bonnet D, Schmaltz AA, Feltes TF (2005) “Infection by the respiratory syncytial virus in infants and young children at high risk." Cardiol Young 15: 256-265.

23. Skoulakis CH, Tigiroglou E, Gkarelis K, Klapsa D, Damani A, et al. (2008) "Level of Streptococcus pyogenes in patients with recurrent tonsillitis and tonsillar hypertrophy." Scand J Infect Dis 40: 899-903.

24. Dalal I, Roifman CM (2015) " Immunity of the newborn." Literature review current through.

25. Nitsch-Osuch A, Choroszy-Król I, Kuchar E, Korzeniewski K, Życińska $\mathrm{K}$, et al. (2015) "Microbiological spectrum and susceptibility pattern of clinical isolates from the neonatal unit in a single medical center." Adv Clin Exp Med 24: 15-22.

26. Mayanskiy N, Alyabieva N, Ponomarenko O, Lazareva A, Katosova L, et al. (2014) "Serotypes and antibiotic resistance of non-invasive Streptococcus pneumoniae circulating in pediatric hospitals in Moscow, Russia." Int J Infect Dis. 20: 58-62.

27. Lu TL, Cao Y, Zhou LX, Huang MJ, Xu X, et al. (2015) " The Antimicrobial Resistance Analysis of 73 Strains of Streptococcus pneumoniae Isolated from Infant Respiratory Tract." Sichuan Da Xue Xue Bao Yi Xue Ban 46: 417-421.

28. Alzeer AH (2009) "Respiratory tract infection during Hajj." Ann Thorac Med 4: 50-53. 